\title{
Isolated Extranodal Natural Killer T-Cell Lymphoma of the Orbit in a Young Patient: Complete Regression with the SMILE Regimen
}

\author{
Rachna Meel ${ }^{a}$ Rebika Dhiman ${ }^{a}$ Meenakshi Wadhwani ${ }^{a}$ Seema Kashyap ${ }^{b}$ \\ Sanjay Sharmac Ajay Gogiaa, d \\ ${ }^{a}$ Oculoplasty and Ocular Oncology Services, ${ }^{b}$ Ocular Pathology Services and ${ }^{c}$ Ocular Radiology Services, Dr. R.P. \\ Centre for Ophthalmic Sciences, All India Institute of Medical Sciences, and ${ }^{d}$ Dr. B.R. Ambedkar Institute Rotary \\ Cancer Hospital, New Delhi, India
}

\section{Key Words}

Natural killer T-cell lymphoma · Lymphomas · Non-B-cell lymphomas $\cdot$ SMILE regimen

\begin{abstract}
Purpose: Natural killer T-cell lymphoma (NKTL) is a highly malignant tumor that typically arises in the nose and/or paranasal sinuses. Isolated orbital involvement by NKTL is extremely rare, and only few case reports exist in the literature. Herein, we report a case of primary orbital NKTL in a young patient who showed very good response to chemotherapy with the SMILE regimen. Methods: A 28-year-old healthy female presented to us with epiphora and fullness of the lower eyelid. She had previously been diagnosed as orbital pseudotumor and was prescribed oral steroids. However, there was no response to the treatment. She also had a history of recurrent sinusitis. An incision biopsy revealed NKTL. A systemic workup did not reveal any other site of involvement. The patient received 6 cycles of chemotherapy (SMILE) and external beam radiotherapy. Results: The tumor showed complete regression after 2 cycles of chemotherapy. The patient is disease free at $\mathbf{2 4}$ months of follow-up. Conclusion: Reported median survival for primary orbital NKTL is ex-
\end{abstract}

\section{KARGER}

(c) 2016 S. Karger AG, Basel

E-Mail karger@karger.com

www.karger.com/oop tremely poor. This is perhaps the first report of a primary orbital NKTL demonstrating a remarkable treatment response to a newer chemotherapy regimen (SMILE) in combination with radiotherapy.

(c) 2016 S. Karger AG, Basel

\section{Introduction}

Lymphomas involving orbit are generally low-grade B-cell malignancies. Natural killer T-cell lymphoma (NKTL) is extremely rare in the orbit $[1,2]$. It characteristically involves the nose and paranasal sinuses (PNS) with secondary extension into the orbit [1-3]. It is a highly aggressive tumor with poor survival prognosis [3]. Herein, we report a case of primary orbital NKTL in a young patient who showed very good response to chemotherapy with SMILE regimen (steroid, methotrexate, ifosfamide, L-asparaginase and etoposide).

Presented as a physical poster at the 74th Annual Conference of the All India Ophthalmological Society (AIOC) held from February 25-28, 2016, in Kolkata, India. 

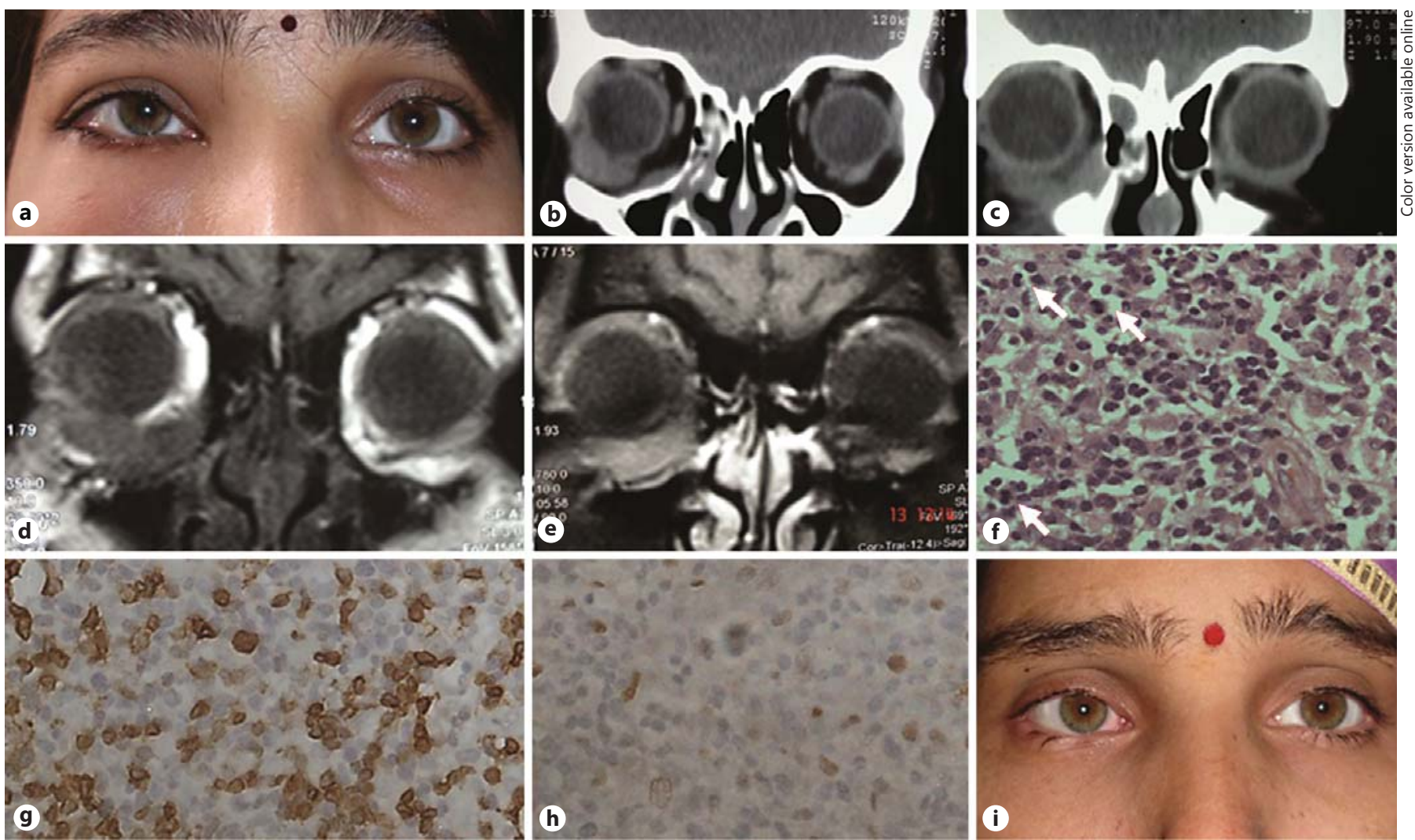

Fig. 1. a Preoperative clinical photograph of the patient showing fullness of the right lower eyelid. b, c Computed tomography scan (coronal section) of the orbit showing a well-defined, hyperdense mass in the inferior orbit that appears to be arising from the orbital floor and medial wall anteriorly and extending toward the globe laterally and posteriorly. d, e Magnetic resonance imaging (coronal section) of the orbit showing a well-defined mass in the

\section{Case Report}

A 28-year-old female patient presented with fullness in the right lower eyelid for the past 6 months. There was a history of right-sided nasal blockage and recurrent maxillary sinusitis, which had been treated conservatively over the last 2 years. In addition, she complained of watering from the right eye for 1 year. A review of her records revealed that she had received treatment with oral steroids for presumed orbital pseudotumor based on imaging findings. However, the patient did not report any improvement in the symptoms. On examination, visual acuity was 20/20 in both eyes. There was fullness of the right lower eyelid, but the overlying skin appeared normal with no signs of inflammation (fig. 1a). There was a mild limitation of movement for depression in the right eye with an upward displacement of the globe. On diplopia charting, diplopia was noted in downgaze. On palpation, the mass in the lower eyelid was nontender and soft-to-firm in consistency and adherent to the orbital floor medially. Slit-lamp examination was noncontributory. The draining lymph nodes were not enlarged. A contrast-enhanced inferior orbit isointense to the muscle on T1-weighted images with good post-contrast enhancement and no mass in the PNS. f Microphotograph showing a cellular lesion with pleomorphic nuclei and numerous mitotic figures (arrows). HE. $\times 200 . \mathbf{g}, \mathbf{h}$ Tumor cells showing positivity for immunohistochemical stains for CD3 and CD56, respectively $(\times 400)$. i Clinical photograph of the patient at the last follow-up.
Ocul Oncol Pathol 2017;3:45-48 DOI: $10.1159 / 000449227$ computed tomographic scan of the orbit and PNS showed a welldefined, hyperdense mass in the inferior orbit arising from the orbital floor medially and extending towards the globe laterally and posteriorly; the inferior oblique muscle could not be seen separately, and there was right ethmoid sinusitis (fig. 1b, c). On a magnetic resonance imaging scan of the orbit, the mass was isointense to the muscle on T1-weighted images, with good post-contrast enhancement. There was no mass in the nose or PNS (fig. 1d, e).

An incisional biopsy was performed. Histopathological examination revealed a cellular lesion with pleomorphic nuclei and areas of necrosis. Tumor cells were arranged around blood vessels. Numerous mitotic figures were also identified (fig. 1f). Immunohistochemistry revealed positivity for CD3 (fig. 1g), cyclin D1 (fig. 1h), CD 56 and Epstein-Barr virus latent membrane protein in the tumor cells. Based on above findings a diagnosis of NKTL was made. Metastatic workup including a whole-body positron emission tomography computed tomography scan and bone marrow biopsy did not reveal any metastasis. The lymphoma was staged as $\mathrm{T}_{2 \mathrm{C}} \mathrm{N}_{0} \mathrm{M}_{0}$ (AJCC classification for ocular and adnexal lympho-
Meel/Dhiman/Wadhwani/Kashyap/ Sharma/Gogia 
Table 1. Literature review of cases of isolated orbital NKTL

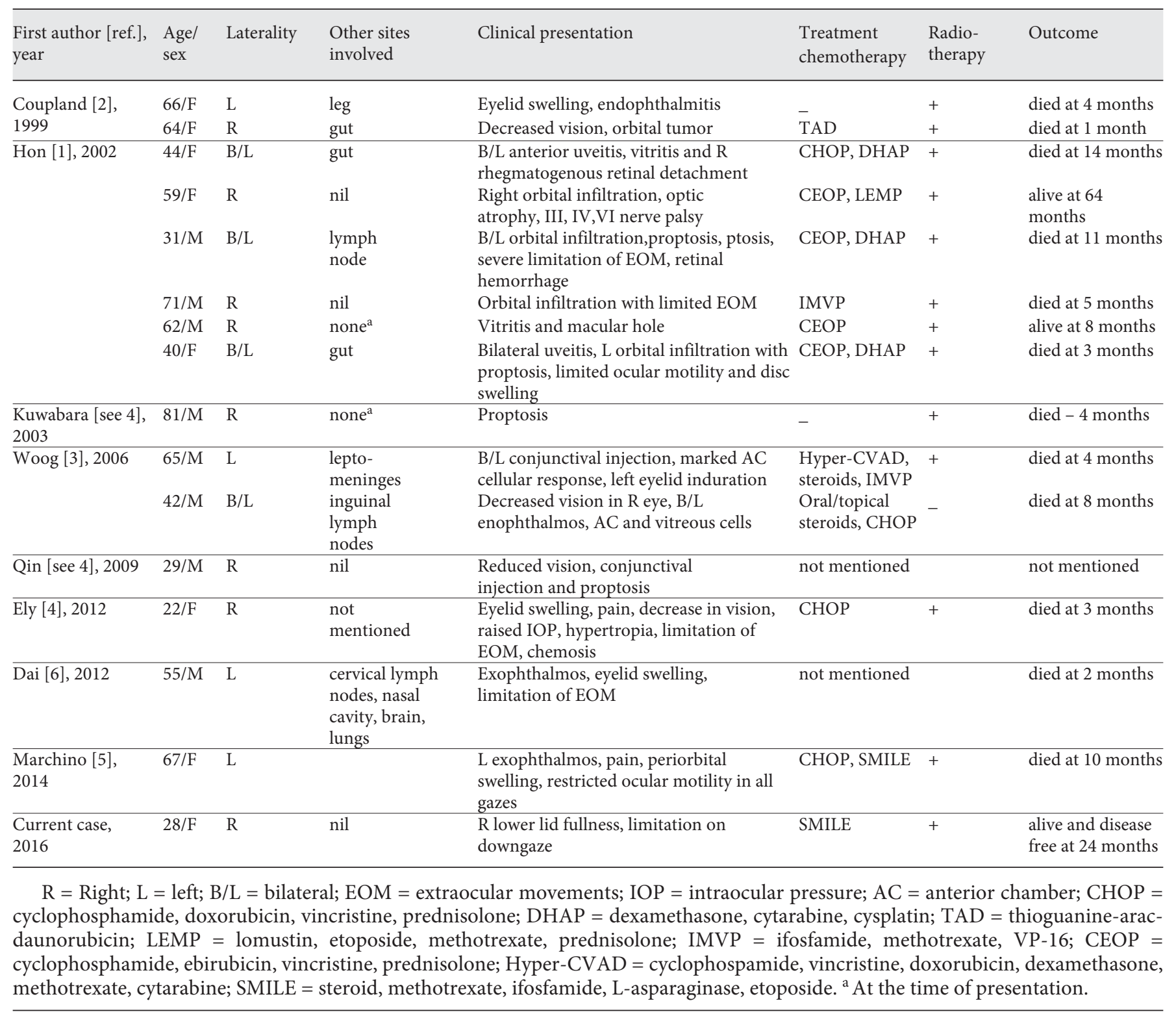

ma). The patient received 6 cycles of chemotherapy (SMILE regimen) and adjuvant external beam radiotherapy to the orbit (46 Gy/25 fractions/ 6 weeks) [4]. The tumor showed complete regression after 2 cycles of chemotherapy. The patient was free of disease at the last follow-up at 24 months (fig. 1i).

\section{Discussion}

Non-B-cell lymphomas represent only $3 \%$ of the primary ocular, orbital and adnexal lymphoproliferative lesions; hence, NKTL is infrequent in the orbit $[2,3]$. Sev- eral studies have reported ophthalmic complications of nasal NKTL, which include uveitis, vitritis, orbital infiltration and even retinal complications speculated to result from severe vitreous inflammation such as macular hole and retinal detachment [1-3]. The intraocular fluid in these cases, however, may not reveal any tumor cells, unlike in high-grade cerebral or ocular B-cell lymphomas [1]. Isolated/primary orbital NKTL of the orbit without evidence of nasal or PNS involvement is extremely rare. Table 1 reviews findings of isolated primary orbital NKTLs reported in the literature [1-6]. The most com- 
mon clinical presentations of primary orbital NKTL that are reported include proptosis, extraocular movement limitation, decreased vision and signs of ocular/orbital inflammation. The rarity of this tumor and inflammatory signs that commonly accompany them make it challenging to identify these tumors early [5]. Orbital NKTL commonly occurs in the fifth decade of life [3]. A younger age at presentation as in the current case can therefore confuse the treating ophthalmologist. Two previously reported cases of orbital NKTL in young adults were misdiagnosed as acute orbital cellulitis [4]. The current case was presumed to be orbital pseudotumor. It is notable that in the current case, there was a history of recurrent sinusitis and intermittent watering in the affected eye before the patient presented with a mass in the inferior orbit. It is possible that this may be unrelated to the orbital disease. On the contrary, we wonder if this could possibly be a manifestation of subclinical involvement of PNS and/or the nasolacrimal system, which was not represented on imaging. Imaging showed evidence of sinusitis but no mass in the nose or PNS. Extranodal NKTL is known for poor survival prognosis with a reported median survival of 12.5 months $[4,5]$. In cases of extranodal NKTL with orbital involvement, the median survival rate is even worse and reported to be only 4 months [1-6]. Several different chemotherapy regimens with/without radiotherapy have been used for treatment of primary orbital
NKTL cases reported in literature. SMILE is a relatively new induction therapy designed by the NK-Cell Tumor Study Group in East Asia, and is reported to show an overall response rate of $81 \%$ in NKTL patients [7]. Marchino et al. [5] used the SMILE regimen as rescue therapy in a 67-year-old patient with primary NKTL and reported good initial response followed by disease progression. Older age is reported to be associated with poor treatment outcome with the SMILE regimen in nasal NKTL [7]. The young age of the patient and a relatively early diagnosis of NKTL may explain the excellent treatment outcome in the current case.

To conclude, primary orbital NKTL is a rare and highly malignant orbital tumor. It may mimic benign clinical conditions. The remarkable treatment response in the current case underscores the need for keeping high clinical suspicion, early biopsy and aggressive treatment with newer chemotherapy regimens such as SMILE.

\section{Statement of Ethics}

Informed consent has been obtained from the patient.

\section{Disclosure Statement}

The authors have no conflicts of interest to disclose.

\section{References}

1 Hon C, Kwok AK, Shek TW, Chim JC, Au WY: Vision-threatening complications of nasal T/NK lymphoma. Am J Ophthalmol 2002; 134:406-410.

2 Coupland SE, Foss HD, Assaf C, Auw-Haedrich C, Anastassiou G, Anagnostopoulos I, Hummel M, Karesh JW, Lee WR, Stein H: Tcell and T/natural killer-cell lymphomas involving ocular and ocular adnexal tissues: a clinicopathologic, immunohistochemical, and molecular study of seven cases. Ophthalmology 1999;106:2109-2120.
3 Woog JJ, Kim YD, Yeatts RP, Kim S, Esmaeli B, Kikkawa D, Lee HB, Korn BS, Punja K, Habermann TM, Colgan JP, Salomao D, Cameron JD: Natural killer/T-cell lymphoma with ocular and adnexal involvement. Ophthalmology 2006;113:140-147.

4 Ely A, Evans J, Sundstrom JM, Malysz J, Specht CS, Wilkinson M: Orbital involvement in extranodalnatural killer T cell lymphoma: an atypical case presentationand review of the literature. Orbit 2012;31:267-269.
5 Marchino T, Ibanez N, Prieto S, Novelli S, Szafranska J, Mozos A, Graell X, Buil JA: An aggressive primary orbital natural killer/T cell lymphoma case: poor response to chemotherapy. Ophthal Plas Reconstr Surg 2014; 30:e131-e134.

6 Dai W, Zhong M, Shen W, Zou K, Bai CG: Natural killer T-cell lymphomaoriginating from the orbit. Chin Med J (Engl) 2012;125: 1677-1680.

7 Kwong YL, Kim WS, Lim ST, Kim SJ, Tang T, Tse E, Leung AY, Chim CS: SMILE for natural killer/T-cell lymphoma: analysis of safety and efficacy from the Asia Lymphoma Study Group. Blood 2012;120:2973-2980. 